\title{
Errata: Reaction-Diffusion Equations for Interacting Particle Systems ${ }^{1}$
}

\author{
A. De Masi, P. A. Ferrari, and J. L. Lebowitz
}

Received December 16, 1986

Expression (4.27a) should be corrected in the following way:

$$
G\left(Y_{\tau}^{\varepsilon}(\phi)\right)-\int_{0}^{\tau} d s L_{\varepsilon} G\left(Y_{s}^{\varepsilon}(\phi)\right)+\int_{0}^{\tau} d s G^{\prime}\left(Y_{s}^{\varepsilon}(\phi)\right)\left[d \mathbb{E}\left(Y_{s}^{\varepsilon}(\phi)\right) / d s\right]
$$

Also, Eq. (4.29) should be corrected as

$$
L_{\varepsilon} Y_{s}^{\varepsilon}(\phi)-\left[d \mathbb{E}\left(Y_{s}^{\varepsilon}(\phi)\right) / d s\right]=\gamma_{1}^{\varepsilon}(\phi, s)+r_{1}^{\varepsilon}
$$

${ }^{1}$ This paper appeared in J. Stat. Phys. 44:589 (1986). 